\title{
POBREZA INFANTIL EN HONDURAS: ANÁLISIS DE PROGRESIVIDAD Y REDISTRIBUCIÓN DE LAS TRANSFERENCIAS DIRIGIDAS A LA NIÑNZ
}

ISSN 2219-6722

ISSNE 2222-2707

Fred Saunders, fred.saunders72@gmail.com - Universidad Nacional Autónoma de Honduras (UNAH)

Sergio Zepeda, Sergiofzepeda@yahoo.es - Universidad Nacional Autónoma de Honduras (UNAH)

Laura Interiano, laurainteriano@hotmail.com - Universidad Nacional Autónoma de Honduras (UNAH)

Seydy Andino, seydy.andino@yahoo.com - Universidad Nacional Autónoma de Honduras (UNAH)

Revista

Economía y

Administración $(E \& A)$

\section{RESUMEN}

Los niveles de pobreza que ha alcanzado Honduras en la última década han derivado un efecto colateral conocido como la pobreza infantil, misma que ha generado fuertes repercusiones en los niños y adolescentes menores de 18 años. Esto a pesar que han existido diversas transferencias dirigidas a la niñez, creadas con el fin único de reducir este efecto negativo y brindar mejores condiciones de vida a los infantes. Por consiguiente, el propósito de la siguiente investigación fue evaluar y analizar el efecto que han tenido las transferencias sociales dirigidas a la niñez en la reducción de la pobreza infantil. Los resultados revelaron que, la contribución de las mismas no ha representado un logro significativo en reducir el total de niños y adolescentes pobres en el país, de igual forma, su impacto es casi inexistente dado a la mala focalización y la falta de políticas públicas complementarias.

Palabras claves: Pobreza Infantil, Transferencias Sociales, Desigualdad, Progresividad, y Redistribución. 


\section{CHILD POVERTY IN HONDURAS: ANALYSIS OF PROGRESSIVITY AND REDISTRIBUTION OF TARGETED TRANSFERS TO CHILDREN}

ISSN 2219-6722

ISSNE 2222-2707

Fred Saunders, fred.saunders72@gmail.com - Universidad Nacional Autónoma de Honduras (UNAH)

Sergio Zepeda, Sergiofzepeda@yahoo.es - Universidad Nacional Autónoma de Honduras (UNAH)

Laura Interiano, laurainteriano@hotmail.com - Universidad Nacional Autónoma de Honduras (UNAH)

Seydy Andino, seydy.andino@yahoo.com - Universidad Nacional Autónoma de Honduras (UNAH)

\section{ABSTRACT}

Poverty levels that Honduras has achieved in the last decade have led a side effect known as child poverty, which he has strong repercussions in children and adolescents under 18 years. This despite various transfers that have been aimed at children, created with the sole aim to reduce this negative effect and provide better living conditions to infants. Therefore, the purpose of this investigation was to evaluate and analyze the effect of social transfers have been aimed at children in reducing child poverty. The results revealed that the contribution of these has not been a significant achievement in reducing overall poor children and adolescents in the country, likewise, its impact is almost nonexistent because of poor targeting and lack of complementary public policies.

Keywords: Child Poverty, Social Transfers, Inequality, Progressivity, and Redistribution.

Revista Economía y Administración (E\&A) 


\section{INTRODUCCIÓN}

En la actual situación de crisis económica por la que atraviesan diversas economías latinoamericanas, la reducción de la pobreza es sin duda el objetivo primordial dentro de la agenda de cada país. En este sentido, diversos gobiernos de América Latina y el Caribe han incluido dentro de sus planes estratégicos, aquellas políticas necesarias para satisfacer las necesidades básicas de cada ciudadano en condiciones de pobreza, por lo que se ha hecho un uso más frecuente de los programas de transferencias sociales condicionados.

Sin embargo, y a pesar de todos estos esfuerzos, muchos países latinoamericanos no han logrado mejorar los índices de pobreza. En consecuencia, se ha desarrollado un efecto colateral conocido como la pobreza infantil, misma que priva a los niños y adolescentes menores de 18 años de los derechos a la salud, la educación, la información, una nutrición adecuada, el agua, el saneamiento básico y la vivienda (Fondo de las Naciones Unidas para la Infancia [UNICEF], Comisión Económica para

Revista Economía y Administración (E\&A) América Latina y el Caribe [CEPAL], 2010).

De ello resulta que, la pobreza que experimentan estos menores se manifieste en tres diferentes etapas: i) La privación, como la falta de condiciones materiales y de servicios esenciales para el desarrollo de todo el potencial de los niños; ii) Exclusión, reflejando el resultado de un proceso injusto a través del cual se les niega la dignidad, la voz y sus derechos, amenazando su desarrollo integral; y iii) Vulnerabilidad, puesto que en una sociedad los más afectados con las crisis son los niños (Christian Children's Fund [CCF], 2005).

Es preciso recalcar que, los niños y adolescentes que hoy en día viven en situación de pobreza, perpetúan su condición hasta la adultez, reproduciendo este patrón a las generaciones subsiguientes. Esto debido a que las privaciones a las que son sometidos generan incapacidad en su desarrollo integral.

Muchas de las personas que hoy en día viven en un entorno de pobreza, son el reflejo de un pasado de privaciones a los servicios y necesidades básicas. Estas privaciones generan un escaso capital humano que limita sus ingresos e impide alcanzar un nivel adecuado de vida, dado que, las necesidades a las que son expuestos los niños y adolescentes generan un efecto permanente en el largo plazo. 
Para el 2011, las estadísticas muestran que un 40.5\% de los niños y adolescentes de Latinoamérica se encontraban en condiciones de pobreza, en donde el $16.3 \%$ representaba la pobreza infantil extrema; en total, la pobreza para ese año afecto a más de 70.5 millones de menores. Según estas estimaciones, Honduras tuvo una incidencia en la pobreza infantil total de $63.1 \%$, lo que nos ubicó como el cuarto país con mayor pobreza infantil, únicamente por debajo de países como Bolivia, El Salvador y Guatemala (CEPAL, 2013).

En vista de lo anterior, resulta de interés conocer si existe una relación bidireccional entre las transferencias sociales dirigidas a la niñez y la pobreza infantil, de igual forma, preguntarse: ¿por qué sigue habiendo un buen número de menores de edad en situación de pobreza?, pese a que se han creado diversos programas con el fin único reducir este efecto; además, evaluar si, ¿es necesario cambiar ciertos criterios de focalización en los programas de transferencias condicionados?; así como discernir, ¿en qué aspectos se está fallando?, y por ende no se está llegando a los más vulnerables.

\section{METODOLOGÍA}

Para el cálculo de la pobreza infantil se usó la Encuesta Permanente de Hogares de Propósitos Múltiples (EPHPM), donde se tomó como referencia los años 2002-2013; considerándose para ello únicamente la población menor de 18 años. De igual forma, se recurrió a la metodología de las Necesidades Básicas Insatisfechas (NBI), misma que es empleada oficialmente por el Instituto Nacional de Estadísticas (INE), y comprende las siguientes necesidades: i) Agua Potable; ii) Saneamiento Básico; iii) Educación; iv) Capacidad de Subsistencia; v) Hacinamiento; y vi) Estado de la Vivienda. En este método, los individuos con una privación de las necesidades antes mencionadas, son considerados pobres relativos, $\mathrm{y}$, con dos o más pobres extremos.

A su vez, para la evaluación de las transferencias se dividió a los hogares con niños y adolescentes menores de 18 años por veintil de ingreso medio per cápita. En donde se calcularon curvas de Lorenz e índices de Gini para los diferentes años, esto con el fin de conocer la distribución y desigualdad en el ingreso, previo a las evaluaciones medidas por el índice de ReynoldsSmolensky ajustado, que mide la forma en que se redistribuyen las transferencias entre los diferentes grupos sociales -en este caso veintiles-; el 
coeficiente de concentración que indica en que veintiles están concentradas la mayor parte de las transferencias; y por último, el índice de Kakwani, mismo que muestra la progresividad o regresividad de las transferencias. Las fórmulas de los indicadores antes mencionados se detallan a continuación:

\subsection{CURVAS DE LORENZ (DISTRIBUCIÓN DEL INGRESO):}

En su cálculo se ordenaron de forma ascendente a los hogares con niños por veintil de ingreso medio per cápita, en el que, el porcentaje acumulado de la población se graficó en el eje horizontal y el porcentaje de ingreso acumulado en el eje vertical. La fórmula utilizada fue la siguiente:

$$
\operatorname{Lx}(v)=\frac{\mathbf{1}}{y} \sum_{0}^{x} x(v) 0<v<1
$$

En donde $\boldsymbol{x}$ es la renta, $\boldsymbol{y}$ es la renta media per cápita, y $\boldsymbol{f}_{\boldsymbol{x}}(\mathbf{v})$ es la distribución de renta por veintil de ingreso.

\subsection{COEFICIENTE DE GINI (DESIGUALDAD EN EL INGRESO):}

Revista

Economía y

Administración

(E\&A)

Este indicador toma valores entre 0 y 1 , donde 0 indica la perfecta igualdad del ingreso entre todos los miembros de la sociedad, mientras que 1 representa la total desigualdad del ingreso en la sociedad. La fórmula que se utilizó fue la siguiente:

$$
G i n i=\mathbf{1}+\frac{\mathbf{1}}{\boldsymbol{n}}-\frac{\mathbf{2}}{n^{2} \boldsymbol{y}} \sum_{i} y i(n+1-i)
$$

En la cual $\boldsymbol{n}$ es el número de veintiles de ingreso, $\boldsymbol{y}$ es la renta media per cápita, $\boldsymbol{i}$ muestra el número de orden del veintil, y $\boldsymbol{y} \boldsymbol{i}$ es la renta por veintil de ingreso. Por lo general, se habla de desigualdad cuando este indicador se presenta entre valores de 0.40 y 0.60 , y, por encima de 0.60 se dice que hay una distribución gravemente inequitativa.

\subsection{REYNOLDS-SMOLENSKY AJUSTADO (REDISTRIBUCIÓN DE LASTRANSFERENCIAS):}

El índice Reynolds-Smolensky ajustado $\prod$ RS, resulta de la resta del Gini sin transferencias $(G X)$, y el Gini con transferencias $(G X+T)$. (Barcena, E. y Imedio, L., 1999). Su fórmula se resume de la siguiente manera:

$$
\prod \mathrm{RS}=\mathrm{GX} \_\mathrm{GX}+\mathrm{T}
$$


En donde, los resultados positivos $(>0)$ indican un efecto redistributivo de las transferencias, y un valor negativo $(<0)$ un efecto no redistributivo, en tanto, si es nulo $\quad(=0)$ resulta que no existe ningún efecto positivo o negativo de las transferencia sobre la renta.

\subsection{PSEUDO-GINI (CONCENTRACIÓN DE LAS TRANSFERENCIAS):}

El Pseudo-Gini o Coeficiente de concentración de las transferencias $(G t)$, es un indicador análogo al coeficiente de Gini, con la diferencia que este se concentra únicamente en medir los montos de las transferencias. Los parámetros de este indicador van desde -1 hasta +1 , en donde un resultado negativo indica que las transferencias son pro-pobre, $y$, por el contrario, un resultado positivo indica que son pro-rico.

\section{5 ÍNDICE DE KAKWANI (PROGRESIVIDAD Y REGRESIVIDAD DE LAS TRANSFERENCIAS)}

Para el cálculo de la progresividad medido por Kakwani (K) fue necesario restar el Gini sin transferencias $(G x)$ con el Pseudo-Gini $(C t)$, tal como se muestra en la siguiente expresión:

$$
\mathrm{K}=\mathrm{Gx}-\text { Pseudo Gini }(\mathrm{Ct})
$$

El valor máximo que resulta de calcular este indicador es $\mathrm{K}=+2$, lo que indica la perfecta progresividad de las transferencias, y caso contrario cuando resulta su valor mínimo $\mathrm{K}=-1$, lo que revela la máxima regresividad (Barcena, E. y Imedio, L. 1999).

\section{CARACTERIZACIÓN DE LA POBREZA INFANTIL}

En todo estudio acerca de pobreza, resulta evidente que la misma afecta tanto a adultos, como a jóvenes y niños. En cambio, la forma en que repercute en cada uno de estos grupos se exterioriza de formas muy disímiles. El deterioro al que son expuestos los niños en su vida por haber crecido en familias pobres, se ve reflejado en la calidad de educación que alcanzan, y por tal, en sus empleos, en la salud, y en general, en su situación social (UNICEF, 2014). La pobreza infantil constituye el ciclo en donde los niños pobres de hoy serán los adultos pobres del mañana, en otras palabras, esto representa un "traspaso generacional de la pobreza". 
Revista

Economía y Administración $(E \& A)$
En Honduras, la evolución que ha presentado la pobreza en el número de niños y adolescentes (véase figura 1)1 ha cobrado una mayor intensidad para finales de esta década, esto producto de la crisis económica mundial de 2008, la cual generó inestabilidad macroeconómica, dejando una marcada inflación de dos dígitos (10.8\%), influenciada por la alza histórica en los precios del petróleo y otros commodities, lo que a su vez profundizó un mayor desequilibrio en la cuenta corriente de la Balanza de Pagos, dejando un déficit de 13.8\% como porcentaje del PIB (Banco Central de Honduras [BCH], 2009).

La crisis de la economía hondureña se profundizó aún más a efecto del conflicto político vivido en 2009; mismo que dejó un ambiente de incertidumbre e hizo disminuir la inversión extranjera y nacional, así como las remesas y reservas internacionales. Este mismo año el PIB decreció en 2.1\%, afectando los sectores de: Construcción, Comercio, Industria Manufacturera y Agricultura, Ganadería, Silvicultura, Caza y Pesca (BCH, 2010).

Esto sin duda ha marcado para los años subsiguientes mayores índices de pobreza en las familias hondureñas, dejando a la población con menores oportunidades de empleo y capacidad de subsistencia. Lo que también resulta perjudicial en aquellos infantes de familias pobres, siendo así que, para el período 2009-2013 el total de menores pobres alcanzó porcentajes arriba del 70\%; lo que demuestra que cada vez son más los niños y adolescentes que no logran en pleno su desarrollo integral.

Es preciso recalcar que las crisis generan una mayor vulnerabilidad en los menores de edad, dado que afectan de manera directa su desarrollo integral. Por esa razón, es preciso que el Estado y la sociedad intervengan y garanticen un mayor cuidado y protección a los infantes, ofreciendo transferencias que se distribuyan de manera eficiente y equitativa: dirigidas a lograr el pleno desarrollo de los menores en situación de pobreza, esto de acuerdo a las necesidades que demanden las familias.

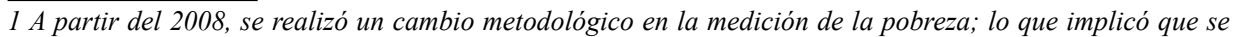
dejaran de emplear factores de ajuste, y asi tomar a detalle los otros ingresos. Esto para que se incluyeran todos los hogares que reportaban ingresos y no exclusivamente los que reportaban un ingreso laboral. Por consiguiente, dicho cambio no afecta la medición de la pobreza por el método de las NBI, dado que la capacidad de subsistencia no tiene que ver con los ingresos en sí, sino con la existencia en el hogar de un perceptor de ingresos. Es decir, con esta dimensión únicamente se quiere saber si el jefe del hogar está ocupado, no cuánto es su ingreso. 


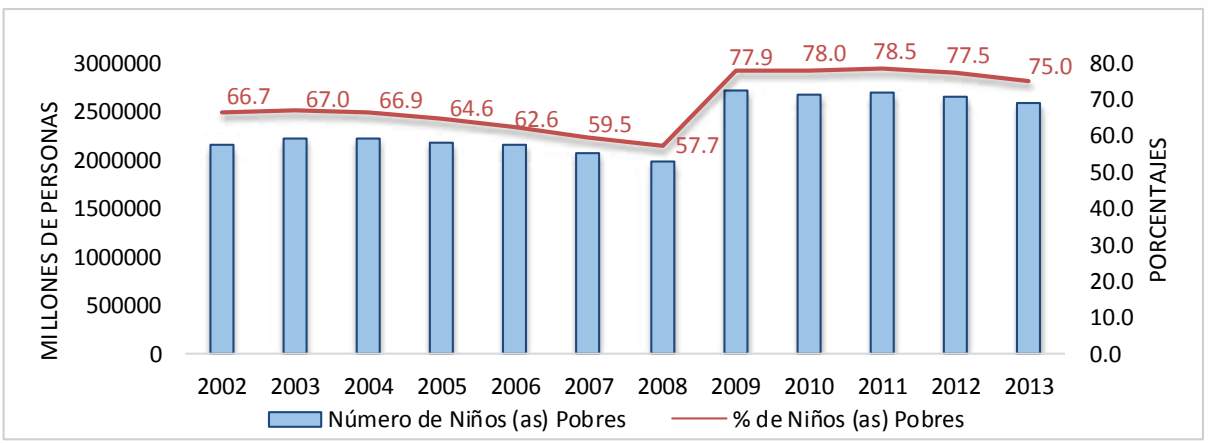

Figura 1: Honduras: Evolución de la Pobreza Infantil

Fuente: Elaboración propia con datos de la EPHPM, 2002-2013

Para conocer más a detalle el escenario en que se presenta la pobreza infantil, es preciso entender las diferentes formas de exclusión y desigualdad a las que se ven expuestos miles de niños y adolescentes. Para ello, se muestra la pobreza infantil extrema (véase figura 2), en donde la misma después de situarse en $27 \%(934,473)$ en 2008 , aumentó para 2009 a $40.5 \%(1,412,988)$ -como antes se mencionó, producto de las crisis por las que atravesó el país, por su parte, la pobreza infantil relativa presentó para ese mismo año un porcentaje de $37.4 \%(1,304,944)$. Mientras que los no pobres en 2002 eran un $33.3 \%(1,080,819) ; 35.4 \%(1,195,789)$ en 2005 ; y un $22.1 \%(770,735)$ para 2009. Lo que indica que ha habido un descenso de los no pobres en la última década; de ello resulta que, la pobreza infantil -relativa y extrema- se haya ensanchado aún más.

La recuperación de la economía hondureña ha mostrado inconsistencia durante el período analizado, dado a la moderada recuperación de las economías avanzadas que también fueron afectadas con la crisis económica de 2008. Sin embargo, y, a pesar de la lenta recuperación de la economía nacional se logró reducir la pobreza infantil extrema a $36.2 \%(1,254,865)$ para finales de 2013, esto producto de la reactivación de la economía a través los sectores productivos como ser: Comunicaciones; Agricultura, Ganadería, Caza, Silvicultura y Pesca; Industria Manufacturera; Comercio; e Intermediación Financiera. Dando como resultado un crecimiento de la economía nacional de $2.6 \%$, pero menor en $1.3 \mathrm{pp}$ al presentado en el año 2012, esto por motivo de desaceleración de las exportaciones de bienes y servicios y a la reducción en la inversión privada. ${ }^{2}$

2 Consultar programa monetario 2012-2013 del BCH 
Lo anterior demuestra que, la reducción de la pobreza y la desigualdad va de la mano con el crecimiento de la economía, de manera que, es conveniente que las transferencias vayan acompañadas de políticas complementarias que brinden un mayor dinamismo a la economía, a fin de obtener una mejor distribución del ingreso en los hogares hondureños y reducir el efecto desigual ocasionado por las diversas crisis.

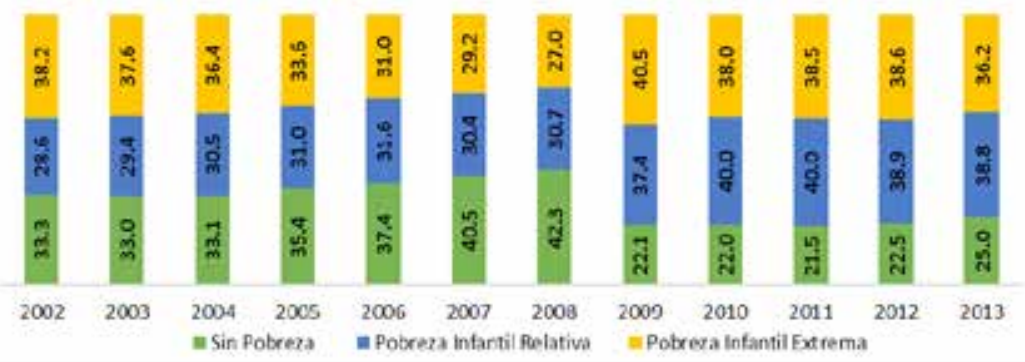

Figura 2: Honduras: Niveles de Pobreza Infantil

Fuente: Elaboración propia con datos de la EPHPM, 2002-2013

Revista

Economía y

Administración

(E\&A)

A modo de profundizar más en el tema pobreza infantil en Honduras, es oportuno detallar las edades, así como el tamaño de las familias y las zonas donde se concentra el más alto número de privaciones entre los menores de 18 años. Esto con la intención de tener un mejor criterio a la hora de elegir los programas que más convienen de acuerdo a las necesidades y características de los niños pobres en el país.

\subsection{POBREZA INFANTIL POR GRUPO DE EDAD}

Entre los grupos de edad analizados -menores de 6 años, entre 6 y 11 años y los de 12 a 17 años- (véase figura 3) son los menores en edad más temprana los que resultan más afectados con las crisis, se apunta que, "1,6 millones de personas mueren cada año de enfermedades diarreicas... atribuibles a la falta de acceso a un agua potable salubre y al saneamiento básico, y un $90 \%$ de esas personas son menores de 5 años, principalmente de países en desarrollo." (Organización Mundial de la Salud [OMS], s.f).

La falta de acceso al agua potable y saneamiento básico está relacionado directamente a la desnutrición y mortalidad infantil; puesto que producto de ello cada vez más niños de temprana edad se enfrentan a enfermedades 
altamente riesgosas como el cólera, diarrea, hepatitis, paludismo, dengue, entre otras. Es por ello conveniente que los infantes crezcan en un ambiente saludable y sin riesgos que perjudiquen su salud y desarrollo.

Por otro lado, se observa que para finales del período de estudio, los pequeños en edad escolar (6-11) son los que exteriorizan la más alta incidencia de pobreza. Asimismo, la negación a la educación representa su mayor obstáculo. Para el (UNICEF y CEPAL, 2010) “existen algunos países centroamericanos, como El Salvador, Guatemala, Honduras y Nicaragua, donde entre el $2 \%$ y el $4 \%$ de los niños en edad escolar nunca ha podido ejercer este derecho".

Para 2002, en Honduras el 25.4\% de la población de 6-17 años no asistía a ningún centro de enseñanza; entre el 2005 y 2009 este porcentaje mejoró, y en promedio un $20 \%$ de dicha población no asistía a ningún centro educativo. Para 2013, el país tuvo una desaceleración y el porcentaje de la población de 6-17 años que no asistía a un centro de enseñanza pasó a representar un 22\%. (EPHPM, 2002, 2005, 2009, 2013).

Las razones que responde a este hecho son múltiples, dentro de las cuales se puede mencionar aquellas que van ligadas al trabajo infantil; la falta de cobertura educacional, principalmente en la zona rural del país; o sencillamente por la falta de recursos económicos que permitan a los padres

Revista Economía y Administración (E\&A) de familia brindarles la educación necesaria a sus hijos. En este sentido, la falta de acceso a la educación conlleva al incremento de la pobreza y la desigualdad en el ingreso, de igual forma, hace que predomine el subdesarrollo y el atraso económico. Es por consiguiente deber del Estado garantizar este derecho ciudadano, haciendo que la misma llegue a aquellas zonas de alta vulnerabilidad, cumpliéndose asimismo con los estándares requeridos para una buena educación.

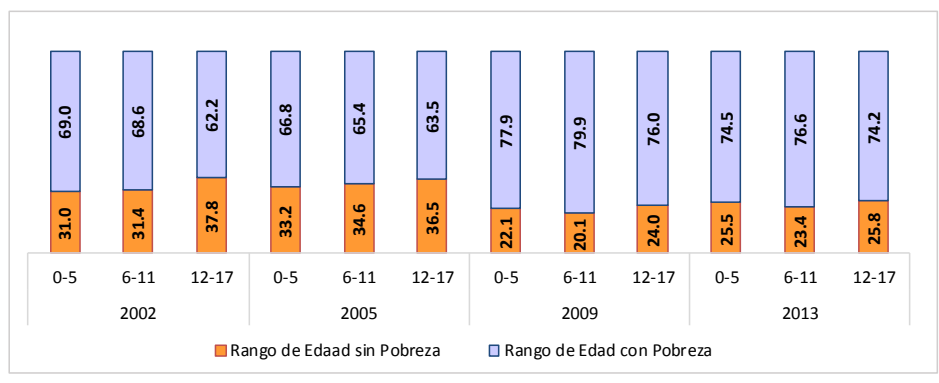

Figura 3: Honduras: Pobreza Infantil por Rango de Edad

Fuente: Elaboración propia con datos de la EPHPM, 2002, 2005, 2009, 2013. 


\subsection{POBREZA INFANTIL POR TAMAÑO DEL HOGAR}

Como se aprecia con claridad en el figura 4 -solo se consideraron los hogares con niños y adolescentes menores de 18 años-, la probabilidad de que un niño sea pobre depende en gran medida del tamaño del hogar, en donde los hogares con un mayor número de hijos presentan una mayor incidencia de pobreza. Lo anterior es un serio problema característico de las familias en la zona rural, principalmente de aquellos lugares más apartados.

Uno de los mayores problemas que enfrentan estos hogares con niños pobres, radica en el estado de sus viviendas, que además de estar construidas con materiales de mala calidad, se encuentran en hacinamiento. En 2013, el $14.6 \%$ de los hogares se encontraban en estado de hacinamiento, donde se estima que eran más de 498,885 las viviendas que estaban en esta situación (EPHPM, 2013).

Revista Economía y Administración (E\&A)

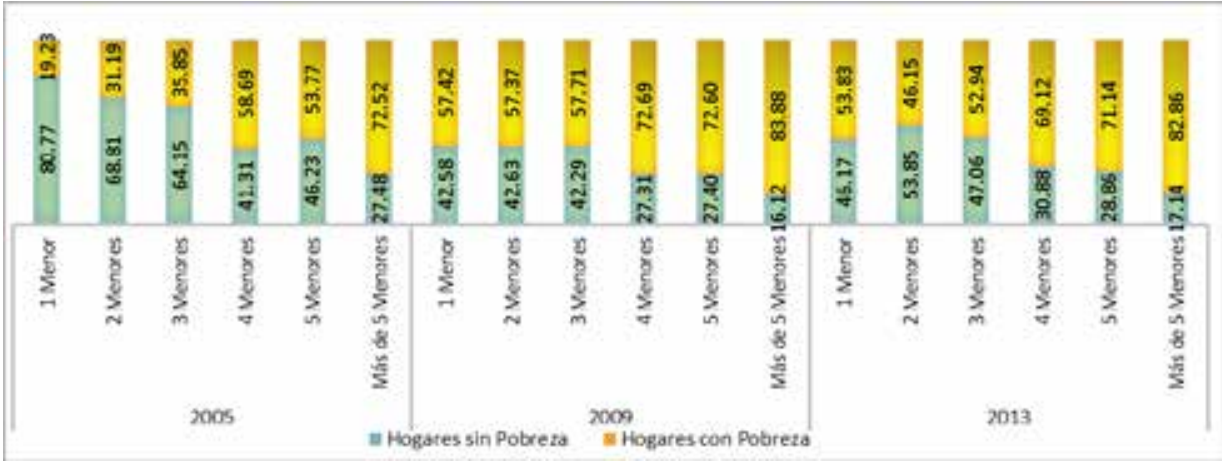

Figura 4: Honduras: Pobreza Infantil por Tipo de Hogar

Fuente: Elaboración propia con datos de la EPHPM, 2005, 2009, 2013

El hacinamiento conlleva a la propagación de enfermedades infecciosas, esto como consecuencia del poco espacio que se comparte en las viviendas, en donde el mismo es usado para diferentes actividades; al estar carentes de las comodidades básicas y la higiene, se produce a una mayor vulnerabilidad frente a las enfermedades. 


\subsection{POBREZA INFANTIL POR ÁREA GEOGRÁFICA}

En la figura 5, resulta de interés ver el porcentaje de niños por departamento que se encontraban en situación de pobreza en 2013. Con base a estos resultados, se puede afirmar que la pobreza infantil se concentra con mayor fuerza en el sur de Honduras, donde la misma alcanzó un nivel superior al 90\%. Igualmente, estos porcentajes revelan como diversos grupos étnicos de niños son afectados, por ejemplo, los Chortis en el departamento de Copán; Lencas en los departamentos de Lempira, Intibucá y la Paz; Tolupanes en Yoro; Pech y Tawahkas en el departamento de Olancho; los Garífunas en Atlántida; y Misquitos en Gracias a Dios -Sin datos disponibles para este departamento-.

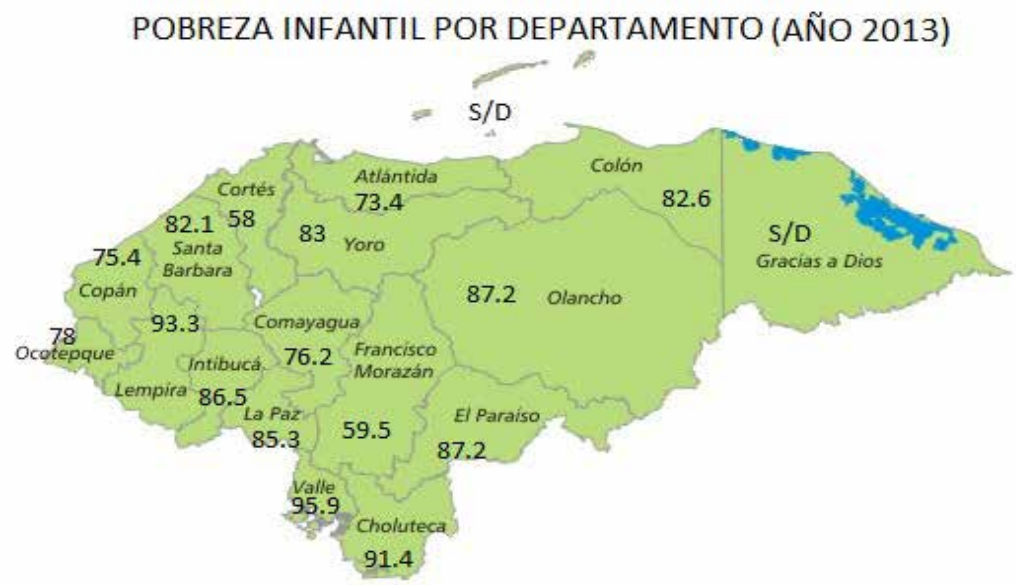

Revista

Figura 5: Honduras: Porcentaje de Pobreza Infantil por Departamento Fuente: Elaboración propia con datos de la EPHPM, 2013

\section{TRANSFERENCIAS SOCIALES DIRIGIDAS A LA NIÑEZ}

Una vez caracterizado la pobreza infantil, resulta provechoso conocer los programas más relevantes destinados a la niñez, del mismo modo, saber qué porcentaje de la producción nacional se está utilizando para mermar el número de niños y adolescentes pobres en el país; esto previo a la evaluación y análisis de las transferencias. 


\subsection{PRINCIPALES PROGRAMAS DE ASISTENCIA SOCIAL DIRIGIDOS A LA NIÑEZ}

Tabla 1: Honduras: Principales Transferencias Monetarias y en Especie Dirigidas a la Niñez

Revista

Economía y

Administración

(E\&A)
Transferencias Monetarios Dirigidos a la Niñez

\begin{tabular}{|c|c|}
\hline \multicolumn{2}{|r|}{ Transferencias Monetarios Dirigidas a la Niñez } \\
\hline $\begin{array}{c}\text { Bono 10,000 } \\
\text { (2010 a la actualidad) }\end{array}$ & $\begin{array}{l}\text { Este programa consiste en el atorgamienta de } \\
\text { transferencias monetarias condicionadas al cumplimiento de } \\
\text { corresponsabilidades, con el propósita de contribuir con la } \\
\text { ruptura del ciclo inter-generacional de la pobreza extrema } \\
\text { relativa y extrema, a través de la creación de oportunidades, } \\
\text { desarrallo de capacidades y competencias en la educación, la } \\
\text { salud y la nutrición de las familias en los hagares en pobreza } \\
\text { extrema y pobreza. }\end{array}$ \\
\hline $\begin{array}{c}\text { Bono Escolar } \\
\text { (1990 a la actualidad) }\end{array}$ & 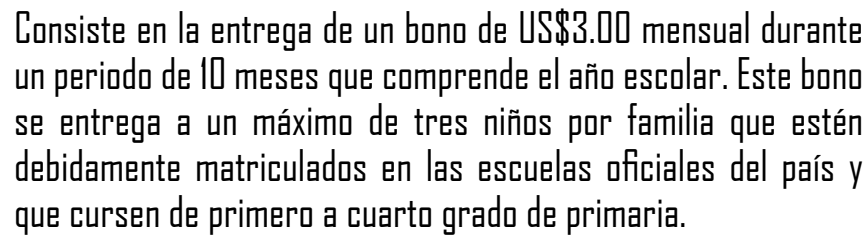 \\
\hline $\begin{array}{c}\text { Materno Infantil } \\
\text { (1990 a la actualidad) }\end{array}$ & $\begin{array}{l}\text { Consiste en la entrega mensual de un bano de US\$3.00 durante } \\
\text { los doce meses del año, beneficianda a la pablación infantil } \\
\text { menar de tres años, niños y niñas discapacitados hasta la edad } \\
\text { de } 12 \text { años y a mujeres embarazadas. Este programa tiene como } \\
\text { objetivo mejorar la dieta alimenticia y aumentar la cobertura de } \\
\text { los servicios básicas de salud. }\end{array}$ \\
\hline \multicolumn{2}{|r|}{ Transferencias en Especie Dirigidas a la Niñez } \\
\hline $\begin{array}{c}\text { Balsón Escolar } \\
\text { (1990 a la actualidad) }\end{array}$ & $\begin{array}{l}\text { Consiste en la datación de un bolsán escolar con sus implementas } \\
\text { escolares a niños y niñas matriculadas en } 10 \text { a } 20 \text { grado. El } \\
\text { objetivo es cooperar con las familias en extrema pobreza en } \\
\text { la cobertura de los gastos asociados con la adquisición de los } \\
\text { materiales y útiles escolares de los hijos. Al mismo tiempo, al } \\
\text { aumenta de matrícula escolar de los más pobres de los pobres. }\end{array}$ \\
\hline $\begin{array}{c}\text { Merienda Escolar } \\
\text { (1990 a la actualidad) }\end{array}$ & $\begin{array}{l}\text { Se creó con el propósito de mejorar la calidad de vida de niñas } \\
\text { y niñas en edad escolar y pre-escolar, reducienda los índices de } \\
\text { desnutrición. El objetivo principal de este programa es ayudar al } \\
\text { logro de las metas del milenio así como aumentar la matrícula } \\
\text { y disminuir los índices de desnutrición ausentisma y deserción. }\end{array}$ \\
\hline
\end{tabular}

Fuente: Elaboración propia con datos de la Secretaría de la presidencia/ Franco, R. (2008) 
Es necesario definir que las transferencias sociales son traspasos de fondos públicos de un grupo social a otro; bajo esta modalidad las mismas pueden clasificarse en dos grupos, el primero de ellos contempla las transferencias monetarias que "son programas sociales que otorgan una transferencia de dinero a las madres de familias en condiciones de pobreza, a cambio del cumplimiento de una serie de condiciones vinculadas a la salud, la educación y la nutrición de sus hijos"3.

El segundo grupo abarca a las transferencias en especie, mismas que "comprenden los bienes y servicios individuales proporcionados a los hogares como transferencias por unidades de las administraciones públicas y las Instituciones sin Fines de Lucro al Servicio de los Hogares (ISFLSH), tanto si se han adquirido en el mercado, como si proceden de la producción no de mercado"4.

De acuerdo al Plan Estratégico de Gobierno 2014-20185, los programas de asistencia social a "favor de la población en extrema pobreza son lideradas por el Bono 10 Mil, el cual desde sus inicios en 2010 hasta agosto de 2013, presenta un total de 462,926 hogares beneficiados a nivel nacional." Asimismo, los demás programas sociales han tenido una corta duración a excepción de Bono Escolar y Bono Materno Infantil.

Actualmente la Secretaría de Desarrollo e Inclusión Social desarrolla el programa de Merienda Escolar, entre otros que forman parte del Programa Vida Mejor. Por otra parte, el Programa de Asignación Familiar (PRAF) sigue brindando la asistencia del Bolsón Escolar, dicho sea de paso, este es uno de los programas en especie a los cuales se le asigna una gran cantidad de presupuesto.

Se enfatiza que los principales programas en monetario y en especie presentados en la tabla 1, responden en mayor medida a las necesidades relacionadas con la educación, la salud y la buena nutrición. No obstante, la pobreza es un problema multidimensional que requiere la atención de todas sus dimensiones. Un mejor enfoque de las mismas, permitirá un mayor progreso en las capacidades que desarrollen los niños y adolescentes, lo que resulta fundamental para lograr el desarrollo sostenido.

3 Consultar: http://ziglablog.com.ar/2010/08/26/programas-de-transferencia-monetaria-condicionada/ 4 Consultar: http://www.eustat.es/documentos/opt 0/tema 222/elem 3557/definicion.html\#axzz3DarX8N47 5 Secretaría de Estado de Coordinación General del Gobierno, (2014). Plan Estratégico de Gobierno 20142018. Documento para la discusión. 


\subsection{GASTO SOCIAL EN INFANCIA O GASTO DESTINADO A TRANSFERENCIAS}

Este gasto incluye únicamente el monto destinado a programas en pro de la niñez, el mismo es ejecutado por las diversas instituciones y Secretarías de Estado con el propósito de erradicar la pobreza y con ello mejorar las condiciones de vida de los menores de edad; en otras palabras, representa el esfuerzo de un gobierno por garantizar que se cumplan los derechos de los niños y adolescentes. Lo interesante en cuanto a este análisis es conocer si dicho gasto tiene una incidencia redistributiva en la renta de las familias.

Como es de apreciar en la figura 6, el gasto social en infancia ha estado por abajo del 1\% del PIB. Según el (UNICEF, 2010), Honduras presentó un gastó por niño de US\$ 108 en 2001, mientras que en 2004 se incrementó a US\$ 121.00, y para 2008 este era de apenas US\$ 199.00 por niño; lo que resultó insuficiente para cubrir las necesidades que presentaban estos infantes, puesto que se requiere de US\$ 384 para cubrir la demanda de servicios que los niños requieren. Con base a ello se alude que, en el país existe una brecha alrededor de $50 \%$ de lo requerido para satisfacer las

Revista Economía y Administración (E\&A) necesidades de la niñez.

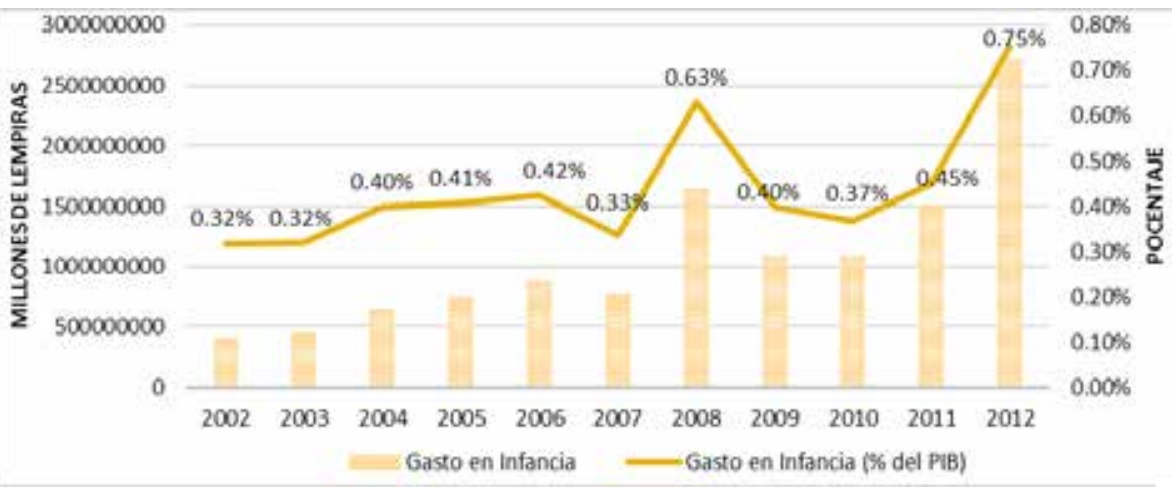

Figura 6: Honduras: Gasto Social en Infancia

Fuente: Elaboración propia con datos de la Secretaría de Finanzas (Dirección General de Presupuesto: departamento de Estadisticas), 2002-2012

En resumen, los fondos destinados en garantizar los derechos de la niñez son insuficientes, mismo que limita el impacto que pudieran tener los programas destinados en mejorar la calidad de vida de los menores; esto a raíz que la mayor parte del ingreso nacional se designa al pago de sueldos y salarios, dejando cada vez un menor presupuesto destinado a la inversión y asistencia social. 


\section{EVALUACIÓN DE LAS TRANSFEREENCIAS}

Para ahondar más en el análisis e incidencia de los programas dirigidos a la niñez, y el efecto que estos tienen en la pobreza infantil, en esta sección se realiza una evaluación de los mismos, empleando para ello indicadores de desigual, distribución, redistribución, concentración y progresividad de las transferencias -consultar metodología-.

\subsection{DISTRIBUCIÓN DEL INGRESO EN HOGARES CON NIÑOS}

A continuación se presenta la distribución del ingreso en los hogares donde reside la población infantil, para ello se han utilizado las Curvas de Lorenz, mismas que muestran la concentración del ingreso en relación a la población de una región para un período determinado. Se dice que, cuanto más lejos este la curva respecto a la recta diagonal, mayor es la desigualdad que predomina entre los distintos grupos sociales.

En consideración de lo anterior, se observan diferentes Curvas de Lorenz para cada año analizado, en donde, la peor distribución del ingreso, dada la cancelación de importantes programas monetarios, fue en 2011 (véase figura 7), lo que implicó que el 20\% de la población tuviera sólo el $2.8 \%$ del ingreso total de la región. También, el $80 \%$ de la población para ese mismo año tan sólo poseía el $40.6 \%$ del ingreso. Sin embargo, en 2013 los niveles de distribución mejoraron de forma muy leve, producto del apoyo a programas monetarios condicionados, lo que resultó que el $20 \%$ de la población ocupará el $3.4 \%$ del ingreso, de igual forma, el $80 \%$ de la población alcanzó el $46 \%$ del total de ingreso (EPHPM, 2011, 2013).

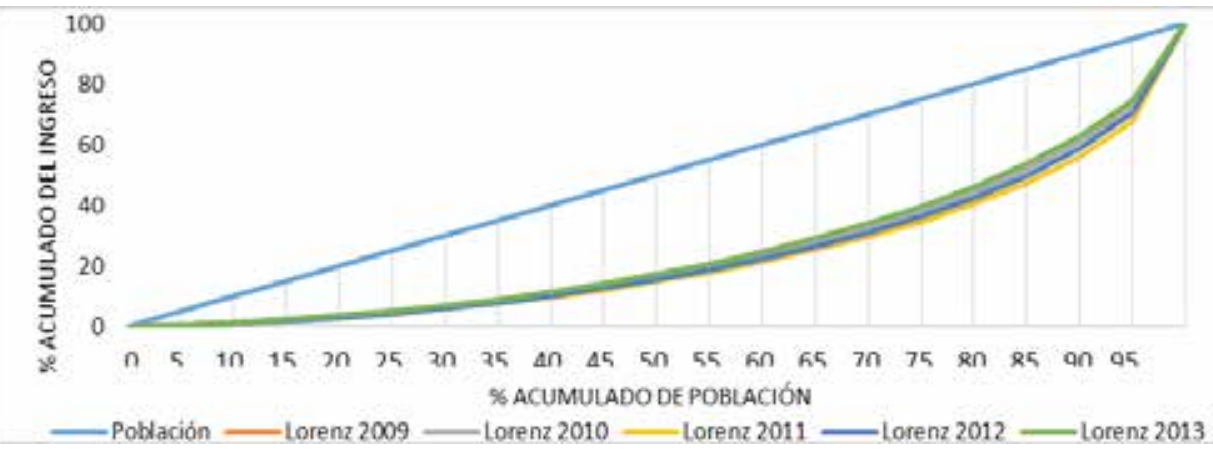

Figura 7: Honduras: Distribución del Ingreso (Curvas de Lorenz) en Hogares con Niños Fuente: Elaboración propia con datos de la EPHPM, 2009-2013 


\subsection{DESIGUALDAD DEL INGRESO EN HOGARES CON NIÑOS}

Análogo a las Curvas Lorenz, se procede a estudiar la desigualdad en el ingreso de los hogares donde residen niños menores de 18 años. Por esa razón se utilizó el coeficiente de Gini; mismo que mide la desigualdad existente en el ingreso de diferentes grupos sociales de un país o región para un determinado período (comúnmente un año).

En relación a la figura 8 se puede percibir que el nivel de desigualdad en el ingreso de los hogares con menores de edad se ha presentado entre valores de 0.40 y 0.50 ; Lo que revela que existe una desigualdad en el ingreso que reciben los diferentes veintiles, ya sea por concepto de rentas, transferencias u otros ingresos. Entre 2009 y 2013, la desigualdad medida por el coeficiente de GINI se redujo, pasando de 0.4979 a 0.4949 . Esta disminución de milésimas puede no ser tan significativa estadísticamente.

De acuerdo con el (Programa de las Naciones Unidas para el Desarrollo [PNUD], 2010) La desigualdad representa un obstáculo para el avance en desarrollo humano, y, dentro de los países más desiguales de América

Revista Economía y Administración (E\&A) Latina con base a la distribución de la renta, Honduras se encuentra entre los primeros lugares, solo por debajo de Haití, Ecuador y Brasil. Esto a diferencia de Uruguay, Costa Rica, Venezuela, Argentina y El Salvador que registran una menor desigualdad.

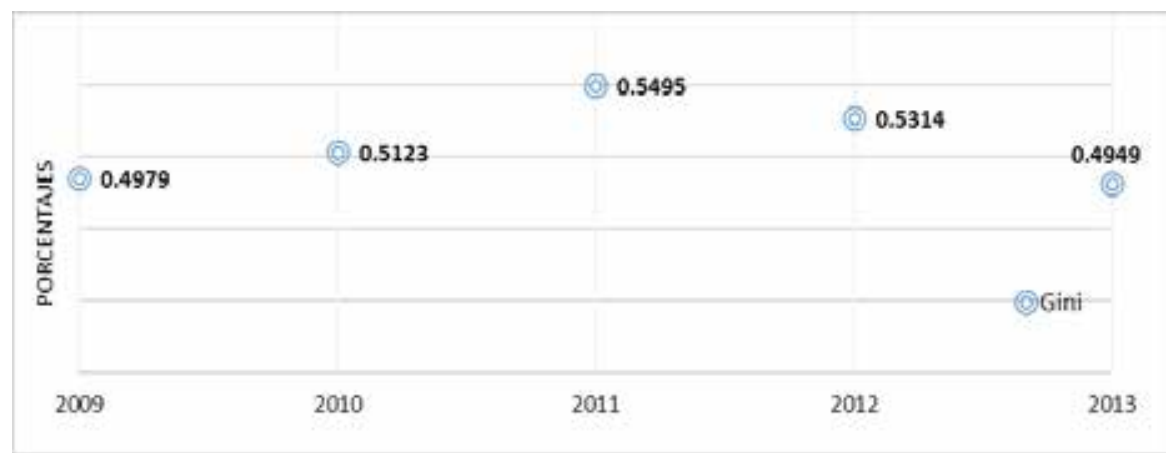

Figura 8: Honduras: Desigualdad del Ingreso (Coeficiente de Gini) en Hogares con Niños Fuente: Elaboración propia con datos de la EPHPM, 2009-2013 


\subsection{REDISTRIBUCIÓN DE LAS TRANSFERENCIAS EN HOGARES CON NIÑOS}

Una vez conocidos los niveles de distribución y desigualdad que existe en los hogares con niños, es de interés ver el grado de redistribución que adquieren las transferencias sociales entre los diferentes veintiles que se ubica la población infantil. En la tabla 2, se pueden apreciar bajos índices obtenidos al calcular Reynolds-Smolensky ajustado, por lo que se deduce que el impacto redistributivo es muy bajo o casi inexistente.

Con respecto a lo anterior, resulta relevante enfocar una mayor atención en el efecto redistributivo que puedan tener las políticas públicas dirigidas a reducir la pobreza infantil, puesto que, un bajo impacto redistributivo de las mismas es un claro resultado de la mala focalización que se tiene al emplearlas. Es por ello que el análisis redistributivo ocupa un gran interés en el campo de la investigación y que en el futuro deberá seguir teniendo para los gobiernos, tanto por su incidencia en reducir la pobreza infantil como por la eficiencia del gasto y la reducción de los altos déficits de recursos públicos.

Tabla 2: Honduras: Redistribución de las Transferencias (Índice de Reynolds-Smolensky Ajustado)

\begin{tabular}{|c|c|c|c|}
\hline Años & GX+T & GX & $\prod \mathbf{R S}$ \\
\hline $\mathbf{2 0 0 9}$ & 0.4979 & 0.5201 & 0.0222 \\
\hline $\mathbf{2 0 1 0}$ & 0.5123 & 0.5460 & 0.0337 \\
\hline $\mathbf{2 0 1 1}$ & 0.5495 & 0.5890 & 0.0395 \\
\hline $\mathbf{2 0 1 2}$ & 0.5314 & 0.5532 & 0.0218 \\
\hline $\mathbf{2 0 1 3}$ & 0.4949 & 0.5281 & 0.0332 \\
\hline
\end{tabular}

Fuente: Elaboración propia con datos de la EPHPM, 2009-2013 


\subsection{CONCENTRACIÓN DE LAS TRANSFERENCIAS EN HOGARES CON NIÑOS}

Entre el período 2009-2013, el Pseudo-Gini o Coeficiente de concentración alcanzó valores entre $0 \mathrm{y}+1$ (véase figura 9); por lo que se concluye que las transferencias a la niñez no tuvieron ese enfoque pro pobre que se necesita para que las mismas sean efectivas e impacten en los estratos más bajos de la sociedad. Lo anterior se deriva de un problema mayormente conocido como la fuga de recursos o tasa de filtración, misma que hace énfasis a aquellas situaciones en donde las transferencias están beneficiando a los no pobres.

Según el Banco Interamericano de Desarrollo (BID, 2012), cuando los programas están limitados a los pobres extremos, es posible que erróneamente se hayan seleccionados beneficiarios que pertenezcan a la categoría de pobres relativos. Por el contrario, si están dirigidos a los pobres relativos, la probabilidad de incluir a los no pobres aumenta. Por consiguiente, para evitar una mayor fuga de recursos se deben considerar los siguientes aspectos: i) El diseño, puesto que no todos los hogares pobres

Revista Economía y Administración (E\&A) viven en las áreas seleccionadas, o satisfacen los criterios demográficos para la elegibilidad; y ii) Errores de exclusión, en el caso que los medios no logren identificar a los pobres como tales. Ampliar la cobertura de las transferencias sin considerar estos aspectos, podría generar una mayor fuga de recursos.

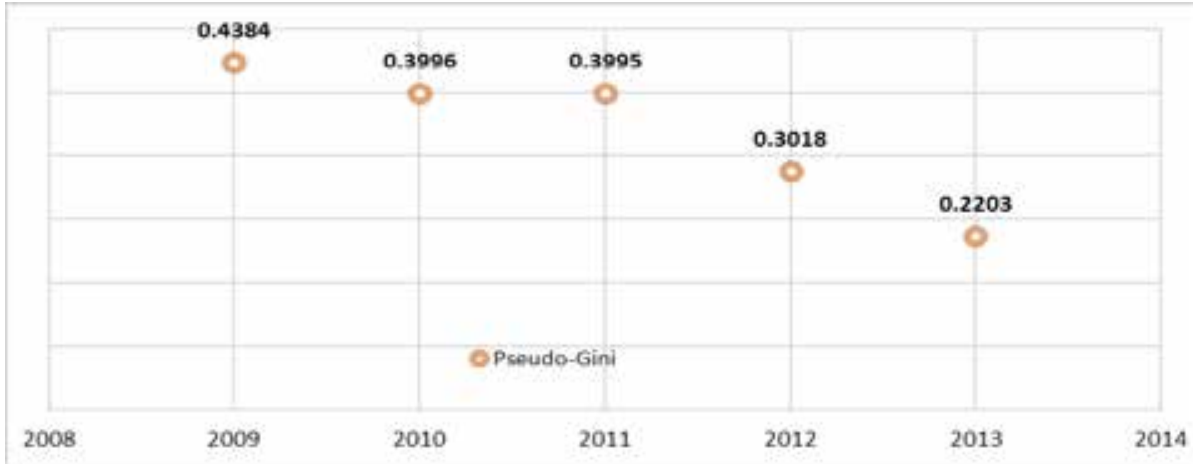

Figura 9: Honduras: Concentración de las Transferencias (Pseudo-Gini) en Hogares con Niños

Fuente: Elaboración propia con datos de la EPHPM, 2008-2013 


\subsection{PROGRESIVIDAD Y REGRESIVIDAD DE LAS TRANSFERENCIAS EN HOGARES CON NIÑOS}

Complementario al Coeficiente de Concentración, está el índice de Kakwani, mismo que captura la progresividad de las transferencias en los hogares. Se entiende que una transferencia es progresiva cuando llega a los estratos más pobres, caso contrario, se considera regresiva. En la siguiente figura se visualiza el nivel en que se encuentra este indicador, como es de apreciar, el mismo presenta valores muy bajos o cercanos a cero, lo que supone que el efecto no es tan progresivo, dicho en otras palabras, es casi nulo.

Esto se deriva por el hecho que los resultados son inferiores a +2 y más cercanos a -1 ; lo mismo se explica por la razón que no hay una clara separación de los pobres extremos y los pobres relativos en la focalización y cobertura de las transferencias, por lo que se deduce que los criterios de elegibilidad no están basados en términos equidad y eficiencia. Dicho sea de paso, esto respalda los resultados mostrados con los indicadores antes mencionados, en donde se concluye que las transferencias no han teniendo una fuerte incidencia en reducir el total de niños y adolescentes pobres en el país.

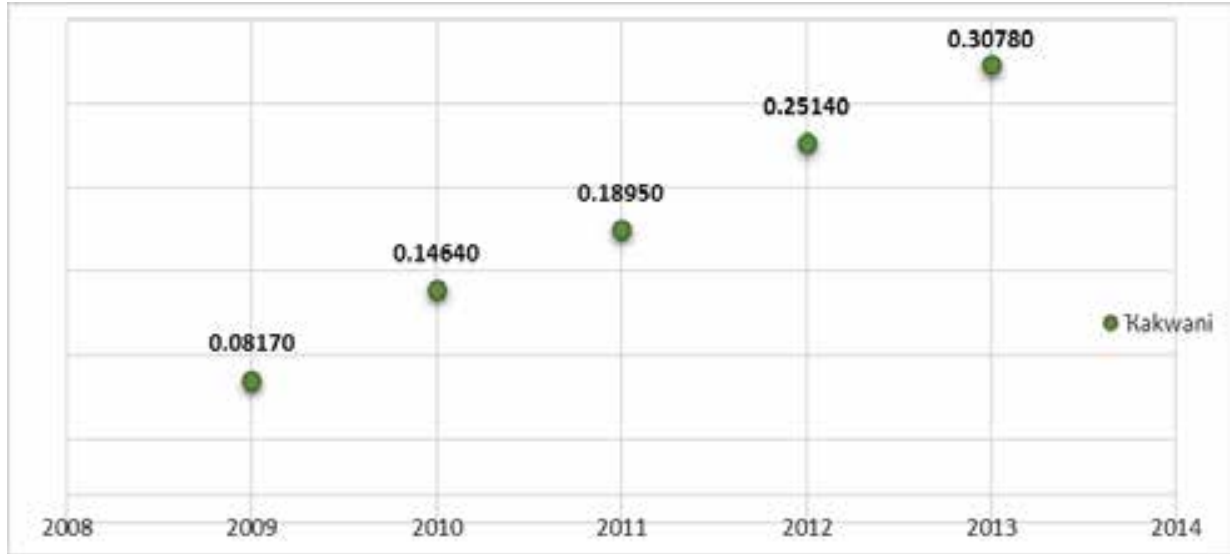

Figura 10: Honduras: Progresividad de las Transferencias (Índice de Kakwani) en los 
La tarea de romper con el traspaso generacional de la pobreza sigue aún pendiente, por lo que resulta preciso revisar la cobertura, así como los criterios de elegibilidad y enfoque que tienen las transferencias; del mismo modo, encaminar los programas en áreas claves para el desarrollo de las capacidades humanas, abarcando todas las dimensiones que comprende la pobreza infantil. Por ende, es conveniente que los programas de asistencia social vayan acompañados de políticas públicas complementarias que garanticen el crecimiento sostenido y generalizado en la población, así como la reducción de la pobreza actual y futura.

\section{CONCLUSIONES}

1. Dentro de los años de estudio la pobreza en niños y adolescentes alcanzó niveles superiores al 70\%; donde la pobreza extrema se situó por encima del $30 \%$, superando en la mayoría de años a la pobreza relativa, en consecuencia, el porcentaje de no pobres se redujo cada vez más para finales de la década. Cabe destacar que, la pobreza en niños se concentra principalmente en la zona sur del país y departamentos fronterizos. Asimismo, la incidencia de pobreza aumenta a medida que crece el número de hijos en el hogar, resultando ser los rangos en edades de 6 y 11 años los más afectados.

2. El gasto social en infancia para los años analizados se situó por debajo del 1\% del PIB; resultando insuficiente para satisfacer la demanda de servicios que requieren los menores de edad. De igual forma, este estuvo mayormente enfocado en las dimensiones de salud, educación y nutrición. En donde los programas de mayor fuerza que aún siguen vigentes son Bono 10.000, Bono Escolar, Bono Materno-Infantil, Merienda y Bolsón Escolar.

3. Los datos demuestran que para el período 2009-2013 hubo una gran desigualdad en los ingresos que percibieron los hogares con niños y adolescentes menores de 18 años. Del mismo modo, el efecto redistributivo que generaron las transferencias en la renta de las familias pobres fue muy bajo, lo que derivo que la concentración de las mismas no se situará en los estratos más pobres de la sociedad; y por ende que su progresividad fuera casi inexistente.

4. Las transferencias no resultaron tener una incidencia positiva en la reducción de la pobreza infantil, producto de ello se explica por la alta fuga de recursos, el escaso presupuesto y falta de políticas complementarias que ayudaran a reducir la desigualdad y aumentar el ingreso de las familias. 


\section{RECOMENDACIONES}

1. Reducir la pobreza y la desigualdad en el ingreso a través de una mejor cobertura y redistribución de las transferencias, focalizando las mismas en términos de equidad y eficiencia, dirigiendo la atención hacía aquellas áreas que comprende la pobreza infantil, a modo que se beneficien directamente los receptores ubicados en los primeros estratos de la sociedad.

2. Con el fin de hacer más eficiente la política social, se recomienda que los criterios de elegibilidad de los programas de transferencias sociales se dirijan directamente a aquellos hogares que se encuentran en situación de extrema pobreza y con niños en edades menores de 12 años, puestos que estos son los más afectados en cuanto a pobreza infantil se refiere.

3. Es preciso que exista un sistema de monitoreo transparente que permita realizar evaluaciones rigurosas acerca de la forma en que se desarrollan los programas de asistencia social orientados a la niñez, además de dar a conocer a la sociedad la información pertinente a los programas; dado que este es un trabajo colectivo entre Sociedad y Estado.

\section{PROPUESTAS}

1. Es de considerar que para aumentar el impacto de las transferencias sociales, las mismas deben de ir acompañadas por políticas complementarias que reduzcan la desigualdad y fortalezcan el efecto redistributivo en la población. Es por ello que se considera que el sistema tributario debe estar orientado hacia una recaudación más progresiva y equitativa, acentuando la política fiscal desde el enfoque pro pobre; esto con el fin de mejorar el nivel de renta en los hogares de menor ingreso y reducir la desigualdad.

Con base a lo anterior se propone: i) fortalecer la recaudación tributaria mediante una gestión más eficaz y transparente que evite la evasión fiscal; ii) ampliar la base tributaria a través de un proceso gradual en el cual todas las empresas -incluyendo el sector informal de la economía- tributen de forma más ecuánime; y iii) revisar el renglón de las exoneraciones fiscales y reconsiderar aquellos regímenes que no contribuyen al desarrollo del país. 
2. Otra política complementaria que contribuiría a mejorar el efecto de las transferencias sociales, es la relacionada al empleo, esto debido a que los altos índices de pobreza en el país se deben en buena medida a la falta de oportunidades que tienen los jefes de hogar en el mercado laboral. Sin embargo, la situación de crisis y represión por la que atraviesa la economía hondureña limita la generación de nuevos empleos.

En vista de lo anterior se propone que, la generación de nuevos empleos sea impulsada por la inversión extranjera directa, esto a través de: i) una mejora de la imagen de país mediante el descenso de los altos índices delincuenciales; ii) lograr una política económica estable y orientada a alcanzar un crecimiento económico sostenido y equitativo; iii) garantizar la seguridad jurídica; y iv) la mejora de la infraestructura vial y portuaria.

Revista Economía y Administración (E\&A)

\section{REFERENCAS BIBLIOGRAFICAS}

Banco Interamericano de Desarrollo, BID (2012). The growth of conditional cash transfers in Latin America and the Caribbean: did they go too far?: Marco Stampini y Leopoldo Tornarolli.

Banco Central de Honduras, BCH $(2009,2010)$. Programa Monetario. Recuperado de: http://www.bch.hn/programa monetario.php

Bárcena Elena, Imedio, Luis J, (1999). Progresividad y efecto redistributivo de las transferencias públicas en Andalucía: Revista de Estudios Regionales, vol. 01, pág. 15-40.

Christian Children's Fund, CCF (2005). Understanding Children's Experience of Poverty: An Introduction to the DEV Framework. Virginia, EEUU: Daniel Wordsworth, Mark McPeak, \& Thomas Feeny.

Comisión Económica para América Latina y el Caribe, CEPAL (2010). Panorama Social de América Latina. Santiago, Chile: Martín Hopenhayn, Ana Sojo, Xavier Mancero, Ernesto Espíndola, María Nieves Rico, Guillermo Sunkel y Pablo Villatoro.

Fondo de las Naciones Unidas para la Infancia, UNICEF (2014). Políticas Públicas Para reducir la Pobreza infantil en España. Barcelona, España: Olga Cantó Sánchez y Luis Ayala Cañón. 
Fondo de las Naciones Unidas para la Infancia, UNICEF y la Comisión Económica para America Latina y el Caribe, CEPAL (2010). Pobreza Infantil en América Latina y el Caribe: María Nieves Rico, Enrique Delamónica, Ernesto Espíndola, Maren Jiménez y Alejandra Silva.

Fondo de las Naciones Unidas para la Infancia, UNICEF (2010). La Infancia en Honduras, Análisis de Situación 2010. Tegucigalpa, MDC.

Franco, R. (2008). Protección Social en Honduras: El Papel de los Programas de Transferencias Condicionadas: PRAF I, II y III. IFHC/ CIEPLAN. 2008. São Paulo, Brasil, e Santiago de Chile. Recuperado de: http://www.plataformademocratica.org/Publicacoes/223.pdf

Instituto Nacional de Estadísticas, INE (2002-2013). Tegucigalpa, Honduras. Encuesta Permanente de Hogares para Propósitos Múltiples (EPHPM). Base de datos disponible en biblioteca del INE.

Organización Mundial de la Salud, OMS (s.f). Agua potable salubre y saneamiento básico en pro de la salud. Recuperado de: http://www.who.int/water_sanitation_ health/mdg1/es /

Programa de las Naciones Unidas para el Desarrollo, PNUD (2010). Informe Regional sobre Desarrollo Humano para América Latina y el Caribe 2010: San José, Costa Rica.

Secretaria Del Despacho Presidencial (s.f.). ¿Qué es el Bono 10 Mil? [Mensaje en un blog]. Recuperado de: http://bonodiezmil.blogspot. com/p/que-es-el-bono-10-mil.html

Este artículo fue seleccionado de trabajos presentados en la Segunda Conferencia Internacional sobre Economía, Administración y Tecnología, evento organizado por el Consorcio Economía, Administración y Tecnología (CEAT). El documento original ha seguido el proceso de revisión estándar de la Revista Economía y Administración (E\&A). El proceso fue dirigido por el Ing. Marvin Aguilar (CEAT-2014) y supervisado por el PhD. Jorge Flores Silva, MSc. Manuel Flores Fonseca y PhD. Jesús Argueta Moreno (Editores de E\&A). 